\title{
ENVOLVENTE ARQUITECTÓNICA: UN ESPACIO PARA LA SOSTENIBILIDAD
}

\author{
ARChitectural ENVELOPE: A SPACE FOR SUSTAINABILITY
}

Manuel Ricardo González Vásquez

Luis Fernando Molina-Prieto**

Recibido: 17 de marzo de 2017

Aceptado: 23 de junio de 2017

\section{Resumen}

El artículo estudia las envolventes arquitectónicas y su importancia en la sustentabilidad urbana y arquitectónica. Se revisa de manera sucinta la evolución histórica que, gracias al descubrimiento del petróleo en el siglo XX, hizo a un lado las técnicas bioclimáticas pasivas que fueron desarrolladas por la arquitectura vernácula de todos los continentes. El objetivo del artículo es hacer evidente la importancia de la envolvente arquitectónica en términos de habitabilidad, a partir de tres variables: iluminación natural, materiales y eficiencia energética. Como metodología se hizo una revisión sistemática de dos tipos de documentos: i) los concernientes a la participación de la arquitectura y la ciudad en el fenómeno del calentamiento global; y ii) los vinculados al concepto contemporáneo de envolvente arquitectónica. Se concluye que la envolvente debe diseñarse con rigor y detalle, para que contribuya a los actuales objetivos de mitigación del calentamiento global.

Palabras clave: envolvente, diseño, materiales, clima, sustentabilidad.

\section{Abstract}

The article studies the architectural envelopes and their importance in urban and architectural sustainability. It briefly reviews the historical evolution that, thanks to the discovery of oil in the twentieth century, put aside the passive bioclimatic techniques that were developed by the vernacular architecture of all continents. The aim of the article is to highlight the importance of the architectural envelope in terms of the habitability, based on three variables: natural lighting, materials and energy efficiency. As a methodology a systematic review of two types of documents was made: i) those concerning the participation of architecture and the city in the phenomenon of global warming; and ii) those linked to the contemporary concept of architectural envelope. It is concluded that the envelope should be designed with rigor and detail, to contribute to the current mitigation objectives of global warming.

Keywords: envelope, design, materials, weather, sustainability.

\footnotetext{
* Arquitecto. Docente investigador, Facultad de Arquitectura, Fundación Universidad de América, Bogotá, Colombia. Grupo de investigación: Territorio y Habitabilidad. ORCID: https://orcid.org/0000-0002-3414-9976. manuel.gonzalez2@ profesores.uamerica.edu.co

${ }^{* \star}$ Arquitecto, M.Sc en Gestión Ambiental (c). Docente investigador, Facultad de Arquitectura, Fundación Universidad de América, Bogotá, Colombia. Grupo de investigación: Territorio y Habitabilidad. Editor de Arkitekturax Visión FUA. ORCID: http://orcid.org/0000-0002-3039-427X. Imolinaprieto@ gmail.com
} 


\section{INTRODUCCIÓN}

Desde los más remotos orígenes de la civilización, es decir, desde el Paleolítico superior - 35000 a 10000 años a. C. - los seres humanos han desarrollado una amplia variedad de hábitats para protegerse de las condiciones ambientales y climáticas del entorno. Lo hicieron a partir de los recursos naturales presentes en cada lugar, de manera que las soluciones son muy variadas: cavernas naturales o escavadas en rocas calizas; cabañas primitivas elaboradas con ramas y hojas de árboles; tiendas nómadas confeccionadas con pieles de animales salvajes, entre otras muchas soluciones (Kantner, 2000; Vela Cossío, 1995). Miles de años después, durante el Neolítico -6000 a 3000 años a. C.- y de manera paralela al proceso de sedentarización, las comunidades humanas empezaron a utilizar materiales de construcción más sólidos y durables para sus asentamientos, estableciendo lo que hoy en día se puede considerar como las primeras arquitecturas de las que se tiene noticia: construcciones producidas con materiales orgánicos como la madera, el bambú o la guadua; e inorgánicos, como la piedra, la tierra y la arcilla (Feathers, Johnson y Kembel, 2008; Stevanovic, 1997; Whitty, 1959). Estas obras, que apelaron especialmente al sentido común de sus constructores, fueron elaboradas a partir de conocimientos básicos y empíricos, y tienen dos importantes elementos en común: i) el uso de los materiales que ofrecía el entorno inmediato; y ii) una profunda armonía con las condiciones climáticas del lugar.

En lo concerniente a la sustentabilidad, cabe anotar que durante siglos, las diferentes comunidades y culturas de los cinco continentes aprovecharon de manera racional los recursos naturales que les ofrecía el entorno, y construyeron sus arquitecturas dando respuesta al clima. Pero a partir de la Revolución Industrial — que surgió a finales del siglo XVIII—, esta situación se modificó, dando paso a la explotación irracional de los recursos naturales para la producción de productos de consumo masivo, dando paso a lo que más tarde se conocería como la crisis ecológica mundial, que se manifestó a nivel global durante la década de 1970, y que hasta la fecha, no ha logrado superarse (Bennett, 2016; Fathy, 1986; Jie, 2014).

La arquitectura, por su parte, mantuvo durante muchos siglos respeto y armonía con las condiciones climáticas de cada lugar, recurriendo para ello a los sistemas de ventilación, refrigeración y calefacción natural que evolucionaron a partir de la arquitectura vernácula. Pero, en los albores del siglo XX, y por efecto del descubrimiento del petróleo, la sabiduría vernácula que comprendía innumerables técnicas pasivas para la regulación de la temperatura en las edificaciones, fue abandonada (Butti y Perlin, 1985). Surgió entonces el movimiento moderno, que para generar confort térmico en sus edificaciones, se apoyó — salvo contadas excepciones-, en los sistemas activos de acondicionamiento lumínico y térmico, es decir: aire acondicionado, calefacción, ventilación e iluminación artificiales, todos ellos alimentados con energía eléctrica generada a partir de combustibles fósiles (Ackermann, 2013). Energía que, en la década de 1950 era muy barata, y lo más importante: no había manifestado los efectos contaminantes y devastadores que dos décadas después se harían evidentes (Brown, Flavin y French, 2000; Färe, Grosskopf y Tyteca, 1996).

Es bien sabido que, durante las últimas décadas del siglo XX y las primeras del XXI, el uso masivo, desproporcionado y agotador de los recursos naturales ha sido una constante en todos los rincones del planeta. Lo que no se sabe abiertamente, al menos en el campo de la arquitectura - porque no se ha reflexionado sobre ese tema en profundidad-, es que gran parte de esa sobreexplotación se debe a la enorme demanda de materiales y energía que requieren los asentamientos humanos, que deben alojar a una población que en 2015 ya superaba los siete mil millones de habitantes (UNFPA, 2015ํ). Todos ellos construidos a partir de paradigmas arquitectónicos que, en su gran mayoría, no responden por su diseño a las condiciones climáticas del lugar —como

\footnotetext{
${ }^{1}$ Fondo de Población de las Naciones Unidas (UNFPA, sigla en inglés).
} 
lo hacían las viviendas del Paleolítico, el Neolítico y la tradición arquitectónica vernácula-, y en consecuencia, requieren enormes cantidades de energía para hacerlo de manera no natural, o sea, mediante sistemas artificiales (Heywood, 2015; Pérez-Lombard, Ortiz y Pout, 2008; Santamouris et ál., 2001).

Esta situación, además de generar altos niveles de contaminación ambiental y propiciar la destrucción de muchos ecosistemas, es totalmente insustentable. Algunas cifras lo evidencian. Las ciudades ocupan el $2.8 \%$ de la superficie terrestre, sin embargo, consumen cerca del $75 \%$ de la producción mundial de recursos de todo tipo, lo cual ha llevado a que en los últimos 40 años, se dupliquen las emisiones de $\mathrm{CO}_{2}$ (Alchapar, Correa y Cantón, 2014). Las ciudades consumen algo más del $40 \%$ de la energía que se genera a nivel mundial, por ende, tienen una gran responsabilidad en la contaminación por efecto de $\mathrm{CO}_{2}$ a nivel global. En Estados Unidos, por ejemplo, las edificaciones consumen cerca del $44 \%$ de la energía de ese país, bastante más que lo consumido por la industria (29\%), y por el transporte (27\%). Cabe subrayar que, entre el $50 \%$ y $60 \%$ del total del consumo por edificio en Estados Unidos, es requerido por equipos de iluminación, calefacción y aire acondicionado. Específicamente, los sistemas activos de calefacción y ventilación consumen el 31 \% de la energía, y los de iluminación el $24 \%$. En Colombia la situación no es mejor: en Bogotá el sector residencial consume el 57 \% de la energía, mientras en Barranquilla, es responsable del $41 \%$ del consumo energético de esa ciudad (Rubiano Martín, 2016).

De otro lado, los estudios adelantados por Yan, Shen, Fan, Wang y Zhang (2010), en Hong Kong, evidencian que la construcción de edificios tiene un gran impacto sobre el medio ambiente. Sus resultados demuestran que los procesos constructivos involucran tres fases que consumen mucha energía y generan gran cantidad de gases de efecto invernadero: i) fabricación de materiales de construcción, que generan el 82-87\% de las emisiones contaminantes generadas por esa industria; ii) transporte de materiales, que genera 6-8 \%; y iii) consumo de energía por equipos de construcción que es responsable del 6-9 \% restante. Pero eso no es todo, porque cada edificio durante su ciclo de vida, que se estima en un promedio de 50 años, consume energía y por ende incrementa el calentamiento global. Cabe subrayar que la mayor cantidad de energía requerida por las edificaciones se consume en sistemas de iluminación y climatización artificiales (Dong, Cao y Lee, 2005; Zhao y Magoulès, 2012).

En este contexto, Janda (2011) hace un llamado a los arquitectos, para que lideren la tarea de reducir los consumos de energía en las ciudades, mediante un desarrollo del oficio que busque incrementar la autosuficiencia energética de las construcciones - objetivo estrechamente vinculado con el diseño de la envolvente arquitectónica-, y para que además, tengan en cuenta a los usuarios, haciéndolos partícipes del desempeño energético de los proyectos. De esta manera el oficio del diseño contribuirá con las estrategias de mitigación del cambio climático que con tanta urgencia se requieren a nivel local y global. Un objetivo fundamental para la sustentabilidad de la especie humana, si se considera que la población mundial está en constante incremento, y por ende, las tasas de consumo energético para usos residenciales y comerciales, aumentarán inevitablemente.

Teniendo en cuenta que los factores que generan los mayores consumos de energía en la arquitectura, son los desequilibrios térmicos al interior de la edificación - generados por las ganancias y las pérdidas de calor del objeto arquitectónico- junto con las deficiencias lumínicas, entra en juego un elemento clave: la envolvente arquitectónica, puesto que gracias a la forma y la geometría de sus vanos y ventanas, permite el ingreso de la luz natural a la edificación, y sus materiales de construcción son los encargados de realizar los intercambios de calor entre la arquitectura interior y el entorno (Goia, Perino, Serra y Zanghirella, 2010). De manera que, del diseño de la envolvente, dependen la reducción o el incremento en los consumos de energía de las edificaciones, y en consecuencia, la sustentabilidad de la arquitectura y de la ciudad. 
La envolvente es un concepto contemporáneo que reemplaza e integra dos componentes clásicos de toda arquitectura: la cubierta y las fachadas, los cuales se concebían como simples planos que establecían límites entre los espacios interiores y exteriores de la edificación, y que además, se diseñaban, si bien como partes de un mismo todo, de manera independiente. Por lo contrario, la envolvente es un elemento tan integral y vivo como la piel de un ser humano. Es el dispositivo que aporta protección al objeto arquitectónico, y además, el encargado de los intercambios de aire, luz y calor con el ambiente. Este concepto, que evoca el útero materno, está en gestación, y por tanto debe ser estudiado en profundidad: "Redefinir la piel es por tanto una de las operaciones que más necesita la arquitectura contemporánea” (De Canales, 2001, p. 64).

El objetivo del artículo es evidenciar la importancia de la envolvente arquitectónica en términos de habitabilidad, a partir de tres variables: iluminación natural, materiales y eficiencia energética.

\section{Metodología}

Con base en una primera revisión de la literatura se identificaron dos tipos de documentos vinculados al objeto de estudio: i) los concernientes a la participación de la arquitectura y la ciudad en el fenómeno del calentamiento global; y ii) los vinculados al concepto contemporáneo de envolvente arquitectónica. En segundo lugar, se seleccionaron los documentos de investigación que estudian el tema de las envolventes desde alguno de los siguientes enfoques: iluminación natural, materiales de construcción y eficiencia energética. Finalmente, se tuvieron en cuenta para la elaboración del artículo, los documentos que - desde la arquitectura y desde la industria de la construcción - buscan contribuir con la reducción de emisiones de gases de tipo invernadero.

\section{Resultados}

¿Cuándo surgió la envolvente? Bustamante (2014) realiza un estudio sistemático que, a partir de referentes del Renacimiento, el Barroco, el Neoclásico y el Movimiento Moderno, traza una secuencia evolutiva entre la fachada y la envolvente. En este análisis la cubierta no es considerada parte de la envolvente - solamente las fachadas-, sin embargo, se presentan elementos claves para comprender los orígenes de este concepto contemporáneo. La profundidad de la Envolvente, título del trabajo de Bustamante, hace referencia a los avances del proyecto moderno en este campo, que lograron independizar, primera vez en la historia, la estructura y el cerramiento. Esta innovación permitió diseñar fachadas, ni planas ni gruesas ni macizas, como las del Renacimiento, el Barroco o el Neoclásico, sino profundas y con su propia espacialidad, por lo que generan un espacio intermedio entre los ambientes interiores de las edificaciones, y su entorno. Así se generó una nueva zona del edificio, no bidimensional sino tridimensional - la envolvente-, que se puede definir como el espacio limítrofe entre el adentro y el afuera, y que si se diseña y maneja adecuadamente, puede solucionar y optimizar el desempeño de la edificación, obteniendo eficiencia energética, confort térmico y optimización lumínica, entre otras calidades técnicas y arquitectónicas requeridas por cualquier arquitectura. Bustamante plantea tres categorías de análisis para la envolvente: iluminar, ventilar y mirar, por ser los más utilizados en la arquitectura moderna colombiana.

\section{Iluminación natural}

En lo concerniente a la iluminación natural de los espacios interiores en la arquitectura, Villalba, Pattini y Córica (2012) destacan su relevancia por tres razones: i) ahorros energéticos, reducción del consumo de energías no renovables y contaminantes, y por ende, disminución de la producción de gases de efecto invernadero; ii) activación del ritmo circadiano, lo que es un efecto no-visual de la iluminación natural que beneficia la salud humana; y iii) confort y optimización visual, que con 
la iluminación artificial es imposible de lograr. Subrayan además, que estas ventajas dependen del correcto diseño y planeación de la edificación y de su envolvente, y que para lograrlo se deben tener en cuenta tres escalas: escala urbana, que involucra las características morfológicas urbanas que rodean la edificación, permitiendo o impidiendo el paso de la radiación solar a una determinada fachada, es decir, a un sector o una fracción de la envolvente; escala edilicia, que incluye la orientación, los aislamientos y los patios de la edificación; y componente de paso (la envolvente en sí misma), cuyos elementos compositivos permiten o impiden el ingreso de la radiación solar al interior del edificio. Por su parte Acosta, Campano y Molina (2016) identifican cinco factores que permiten que la luz del día aporte autonomía lumínica a las edificaciones: diseño, forma, tamaño, posición y geometría de las ventanas sobre la envolvente. En la misma línea de investigación, Ávila y Arias (2015) analizan la influencia de la envolvente arquitectónica en la iluminación natural de los espacios interiores. Para ello realizaron una serie de experimentos en el Laboratorio de Ciencias del Hábitat de la Escuela Nacional de Trabajos Públicos, en Lyon (Francia), en los que hicieron un análisis comparativo del índice de transmisión lumínico de cuatro materiales, en cuatro diferentes grados de incidencia lumínica. Los materiales analizados fueron: acrílico especular, acrílico difuso blanco, acrílico bronce, y papel pergamino sobre vidrio común.

En cuanto a la incidencia de la radiación solar sobre la envolvente, y el comportamiento lumínico y térmico que genera en los espacios interiores de la edificación, Evans, Eguía, Pérez y Evans (2003) desarrollaron una metodología de estudio que incluyó: la evaluación del impacto de la radiación solar sobre las fachadas y los espacios interiores; la transmisión de la radiación en los vidrios que hacen parte del diseño de la envolvente; y los niveles de iluminación natural y confort visual de cada uno de los espacios interiores. La metodología fue aplicada al proyecto Ciudad Judicial de Barcelona y Llobregat, y para evaluar los resultados se usaron técnicas bioclimáticas, pruebas con maquetas a escala en el heliodón; simulaciones gráficas por computador y cálculos analíticos. A partir de sus resultados, establecieron recomendaciones específicas que permitieron optimizar el material, las dimensiones y la geometría de cada uno de los elementos que conforman las envolventes de ese proyecto, y además, disminuir las necesidades y demandas de energía eléctrica por parte del complejo arquitectónico, al tiempo que se incrementó la calidad térmica y lumínica de los espacios interiores.

\section{Materiales}

El iglú es un ejemplo perfecto de la vivienda vernácula, donde la respuesta al clima se logra a partir de los materiales locales. En esta arquitectura construida con bloques de nieve, su especial y genial envolvente - una cúpula-, se adapta a condiciones climáticas extremas, y ha permitido la subsistencia de una comunidad nómada, los inuit, en el Círculo Polar Ártico, en países como EE. UU. (Alaska), Canadá, Rusia (Siberia), los países nórdicos (Dinamarca, Noruega, Finlandia, Suecia) y Groenlandia (Bahamon y Cañizares, 2008). En un ambiente hostil, sin tierra ni vegetación y cuyas temperaturas oscilan entre los 10 y 30 grados bajo cero, a partir de una envolvente aerodinámica y cerrada, construida con el único material que tienen a su alcance, los inuit logran, con el calor emitido por una lámpara de aceite de foca y sus propios cuerpos, temperaturas internas hasta de cinco grados centígrados (Moreno, 1993).

Pasando a la arquitectura contemporánea, Alonso, Oteiza y García (2010) reconocen la envolvente como el elemento que gestiona los flujos calóricos que se producen continuamente entre el exterior y el interior de las edificaciones, por lo que de su diseño dependen: la calidad ambiental de los espacios interiores y el potencial ahorro de energía de la edificación. El estudio hace énfasis en los materiales que componen la envolvente, que según sus resultados compromete el $28 \%$ del peso total de la construcción, y contiene el $20.4 \%$ de la energía incorporada en el edificio. Además, 
se analiza la influencia que tienen varios parámetros de diseño de la envolvente - como el tipo de muro de las fachadas y su masa térmica, la relación lleno-vacío, y los materiales seleccionados para su construcción-, sobre la demanda de energía del objeto arquitectónico a lo largo de su vida útil, y por ende, sobre las emisiones de gases de efecto invernadero.

Biswas y Abhari (2014) afirman que la eficiencia energética de los edificios depende en gran medida del material de cambio de fase de la envolvente (PCM por sus siglas en inglés), y que, aunque se encuentran numerosos estudios en la literatura, su aplicación se ha visto limitada por su elevado costo. Para superar esta limitante, experimentaron con ácidos grasos/glicéridos naturales presentes en polietileno de alta densidad, añadiéndolo a los muros exteriores de una edificación ubicada en clima cálido húmedo, y realizando ensayos durante varios meses. Aunque sus resultados son positivos, concluyen que se requieren valoraciones que involucren no solo el comportamiento de los muros de la envolvente con el nuevo material aislante, sino los intercambios que se realizan a través del techo y las ventanas de la edificación. Cabe subrayar que el material de cambio de fase (PCM) de la envolvente arquitectónica ha sido ampliamente estudiado en las dos últimas décadas, por autores como Cabeza, Castell, Barreneche, De Gracia y Fernández (2011); Khudhair y Farid (2004); Kuznik, David, Johannes y Roux (2011); y Sharma, Tyagi, Chen y Buddhi (2009), entre otros.

López, Rubio, Martín, Croxford y Jackson (2015) realizan una investigación sobre la adaptación de las plantas frente a los cambios ambientales, y a partir de ella, plantean el uso de materiales activos para la construcción de las envolventes arquitectónicas. Consideran que los edificios convencionales requieren altos consumos de energía para mantener el confort interior, porque son diseñados para dar una solución estática al clima, que es variable, en lugar de proponer una solución dinámica, como la de las plantas. Para el desarrollo de su propuesta se apoyan en tecnologías de punta, como la impresión 3D multimaterial, las nuevas capacidades de simulación por computador y los avances en la ciencia de los materiales.

A nivel urbano, Alchapar y Correa (2015), a partir de dos conceptos: i) que la envolvente arquitectónica incide de manera directa en la temperatura del ambiente urbano; y ii) que los espacios urbanos tienen su propia envolvente; analizaron el comportamiento térmico de 150 materiales de construcción disponibles en la ciudad de Mendoza, Argentina. Recurrieron al Índice de Reflectancia Solar (IRS) para determinar la incidencia de cada material en la temperatura de la edificación, y en el ambiente urbano. Estudiaron los materiales más utilizados en envolventes arquitectónicas — fachadas y cubiertas - y en la envolvente urbana — superficies de uso vehicular y peatonal-, y para manejar la complejidad de los datos a valorar, clasificaron los 150 materiales en dos grandes grupos: materiales horizontales: tejas y pavimentos; y materiales verticales: revestimientos y pinturas. Finalmente, cada material fue considerado bajo cuatro variables: acabado, textura, forma y color. Concluyeron, que el IRS depende en gran medida del color de la superficie analizada, pero se deben tener en cuenta otros factores, como textura, composición y envejecimiento; y además, que a partir de los materiales de la envolvente arquitectónica y urbana, se pueden generar variaciones hasta de tres grados centígrados en la temperatura del aire de los espacios urbanos, y hasta de doce grados centígrados en la temperatura de las superficies de la envolvente. En un estudio similar, pero orientado a la disminución de las islas de calor urbanas, los investigadores concluyeron que los materiales que se caracterizan por su alto IRS, es decir, los que reflejan la mayor parte de la radiación solar, no se calientan, y cuando están presentes en la envolvente urbana "contribuyen a disminuir la demanda de energía para refrigeración en edificios con aire acondicionado y mejoran el confort de los espacios abiertos, contribuyendo a la sustentabilidad ambiental" (Alchapar et ál., 2013, p. 283). 


\section{Eficiencia energética}

Sadinesi, Madala y Boehm (2011) revisan los elementos que hacen parte de la envolvente, con el objetivo de reducir las demandas de energía por parte de la edificación. Para el análisis fragmentan la envolvente en tres partes: muros, ventanas y cubiertas. Consideran que los muros conforman la fracción predomínate de la envolvente arquitectónica, es la que provee el confort térmico y acústico, y es el elemento que mayor aporte estético hace a la construcción. Tienen en cuenta cuatro tipos de muros porque reducen los consumos energéticos: muros solares pasivos, muros de hormigón ligero, muros ventilados de doble piel y muros con almacenamiento de calor latente. En lo concerniente a los vanos de la envolvente, es decir, las ventanas, los consideran desde dos instancias: i) el tipo de cristal que puede ser doble, holográfico, refractivo, entre otros; y ii) los marcos o soportes, que en muchos casos son responsables de pérdidas de energía de la edificación, por lo que recomiendan los que tienen bajo índice de conductancia. Por último, reconocen las cubiertas como una fracción de la envolvente altamente susceptible a la radiación solar, y a otros fenómenos ambientales, como la lluvia, por lo que de ella depende en gran medida el confort térmico de los usuarios. Analizan ocho tipos de cubiertas: construidas con mampuestos, sistemas de techos ligeros de aluminio, techos ventilados y microventilados, techos abovedados, techos reflectivos, techos verdes, techos fotovoltaicos y techos con aislamientos térmicos.

Desde Argentina Evans y Schiller (2005) analizan el rol de la envolvente en las edificaciones, destacando que deben atender los siguientes factores para ser sustentables: control de pérdidas excesivas de energía en invierno, control de ingreso excesivo de radiación solar en verano, iluminación y ventilación natural de todos los espacios interiores, y disminución del impacto ambiental generado por los materiales utilizados para su construcción. Recomiendan la realización de estudios puntuales, técnicos y ensayos de laboratorio durante y después de terminado el proceso de diseño, para valorar la eficacia de los elementos de protección solar, alcanzar los niveles óptimos de iluminación natural en el interior del edificio, y controlar la temperatura interior en los diferentes momentos climatológicos del año. Además, definen protocolos a seguir para la optimización del proyecto, que permiten alcanzar los objetivos y certificaciones de sustentabilidad que requieren los edificios contemporáneos.

Celis, Escorcia, Díaz, García y Echeverría (2012), desde el enfoque de la eficiencia energética, realizaron una propuesta de diseño de envolvente arquitectónico aplicable a los programas de reconstrucción posteriores al sismo del 2010 en Chile, que azotó las regiones de Maule y Bio-Bio. Hacen énfasis en la importancia de estudiar y comprender las particulares condiciones climáticas que afectan el área de trabajo, por ser un factor vital para el correcto diseño de cualquier proyecto. Analizan además, las características de agrupamiento, la forma y la materialidad de las envolventes, haciendo consideraciones técnicas y económicas y relaciones costo-beneficio para el mejoramiento de las viviendas. Concluyen que la eficiencia energética se logra partiendo de un acertado análisis de las condiciones climáticas, puesto que eso permite una implantación y configuración volumétrica acorde con las necesidades propias del proyecto y las condiciones climáticas del entorno.

Marincic et ál. (2011) realizaron un estudio sobre viviendas de carácter social en proceso de construcción en Hermosillo, Estado de Sonora, México, que se caracteriza por su clima cálidoseco. El trabajo buscaba valorar el grado de adecuación al clima de estas viviendas "mínimas", para evidenciar si contribuían al ahorro de energía destinada a la climatización. Para el estudio se tuvieron en cuenta variables como la orientación del volumen, los materiales y el acabado de la envolvente, los sistemas de control solar, de ventilación, las alturas, el diseño interior y las características de las áreas exteriores. Concluyeron que las viviendas analizadas muestran una muy deficiente adaptación al clima, por lo que no contribuyen a los consumos de energía de ese país, sino que los incrementan. 
Para Ganadeiro, Duarte, Correia y Leal (2013), la forma de la envolvente es la característica de diseño más sobresaliente de una edificación, y tiene una gran incidencia en su desempeño energético. No obstante, consideran que establecer el rendimiento energético de un edificio, durante las primeras etapas de diseño, es una labor bien difícil por la escasez de datos precisos para una simulación o modelación por computador. En consecuencia, proponen una metodología de diseño que involucra la forma de la envolvente en relación al rendimiento energético final. Para ilustrar el protocolo de diseño que proponen, recurren a una de las emblemáticas casas del arquitecto Frank Lloyd Wright en Chicago. Este mismo grupo de investigadores, en otro trabajo (Ganadeiro et ál., 2013), establecieron un nuevo indicador de diseño llamado demanda de energía relacionada con la envolvente (DERE), que facilita a los diseñadores calcular las futuras demandas de energía de una edificación, durante sus fases de diseño iniciales, a partir de la forma y los vanos de la envolvente. En la misma línea de investigación se ubica el trabajo de Méndez, Capozzoli, Cascone y Sassone (2015), puesto que busca establecer indicadores para que los diseñadores tomen decisiones durante las primeras etapas de diseño, que los lleven a desarrollar edificios con alto rendimiento energético. Para su trabajo realizaron simulaciones a partir de un estudio de caso, un edificio de oficinas al que se pretendía minimizar los consumos de energía para calefacción, enfriamiento e iluminación. El edificio se dispuso digitalmente en varios entornos: Palermo, Torino, Frankfurt y Oslo, variando la disposición y geometría de las ventanas, y el grosor de los muros de la envolvente.

Mirrahimi et ál. (2016) valoran el desempeño energético generado por las envolventes, en edificios de gran altura, en Malasia, bajo condiciones climáticas tropicales. Luego de revisar estudios de confort térmico para ese país y describir los componentes de la envolvente arquitectónica, proceden a analizar los sistemas pasivos de refrigeración y control de la temperatura, considerando los siguientes componentes de la envolvente: elementos de ventilación natural, forma, anchura, longitud, altura, orientación, materiales de los muros exteriores, materiales aislantes de la envolvente, materiales de la cubierta, tipos de acristalamiento, proporción muro/vano, y sombras procedentes del exterior. Concluyen que el parámetro de diseño más importante para regular y controlar el confort térmico de una edificación en clima cálido húmedo es la envolvente de la edificación, y resulta crucial porque además, su acertado diseño reduce de manera considerable los consumos de energía.

Sobre la envolvente arquitectónica, y específicamente en el tema de la eficiencia energética, se han realizado numerosas investigaciones, como la de Yang, Lam y Tsang (2008), en China, que valora el desempeño energético generado por los intercambios de calor de la envolvente en los edificios de oficinas de cinco de las ciudades principales de ese país, Harbin, Beijing, Shanghái, Kunming y Hong Kong, las cuales fueron seleccionadas por corresponder con los cinco climas predominantes en China: frío severo, frío, verano cálido e invierno frío, templado, y verano cálido e invierno cálido. O como la de Berggren y Wall, (2013), que busca establecer indicadores fiables que faciliten las labores de diseño de las envolventes arquitectónicas en condiciones de frío severo, en Suecia. O como la de Lei, Yang y Yang (2016), que busca identificar materiales para la envolvente arquitectónica que tengan la capacidad de absorber calor masivo, puesto que desarrollan su trabajo en Singapur, en clima cálido húmedo y con altos índices de radiación solar a lo largo del año. Por solo citar unas cuantas.

Por último, destacamos el trabajo de Guzowski (2013), quien, teniendo en cuenta los efectos del cambio climático, propone que en las escuelas de arquitectura se inicie una formación que exija a los estudiantes bajos consumos energéticos en sus proyectos, e incluso, que generen cero consumo de energía o energía positiva (o sea, más energía que la requerida por el proyecto), de manera que se fomente en la comunidad académica la conciencia, la intuición y las habilidades necesarias para diseñar proyectos energéticamente sustentables. En su trabajo Guzowski explora una serie de módulos didácticos preparados con ese fin, que se basan en el diseño bioclimático, la iluminación 
natural y el confort térmico como temas esenciales para el diseño. Concluye que, de las decisiones de diseño en cualquier proyecto arquitectónico, dependen: i) el confort humano; ii) el consumo de energía; y iii) los impactos ambientales y ecológicos.

En la figura 1, se presenta una síntesis de los aspectos más relevantes a tener en cuenta al momento de diseñar envolventes arquitectónicas, generado a partir de la investigación, y estructurado de acuerdo a las variables de análisis establecidas para abordar el objeto de estudio, es decir: i) iluminación natural; ii) materiales; y iii) eficiencia energética. En la figura 2 se presenta una síntesis gráfica de los resultados de la investigación.

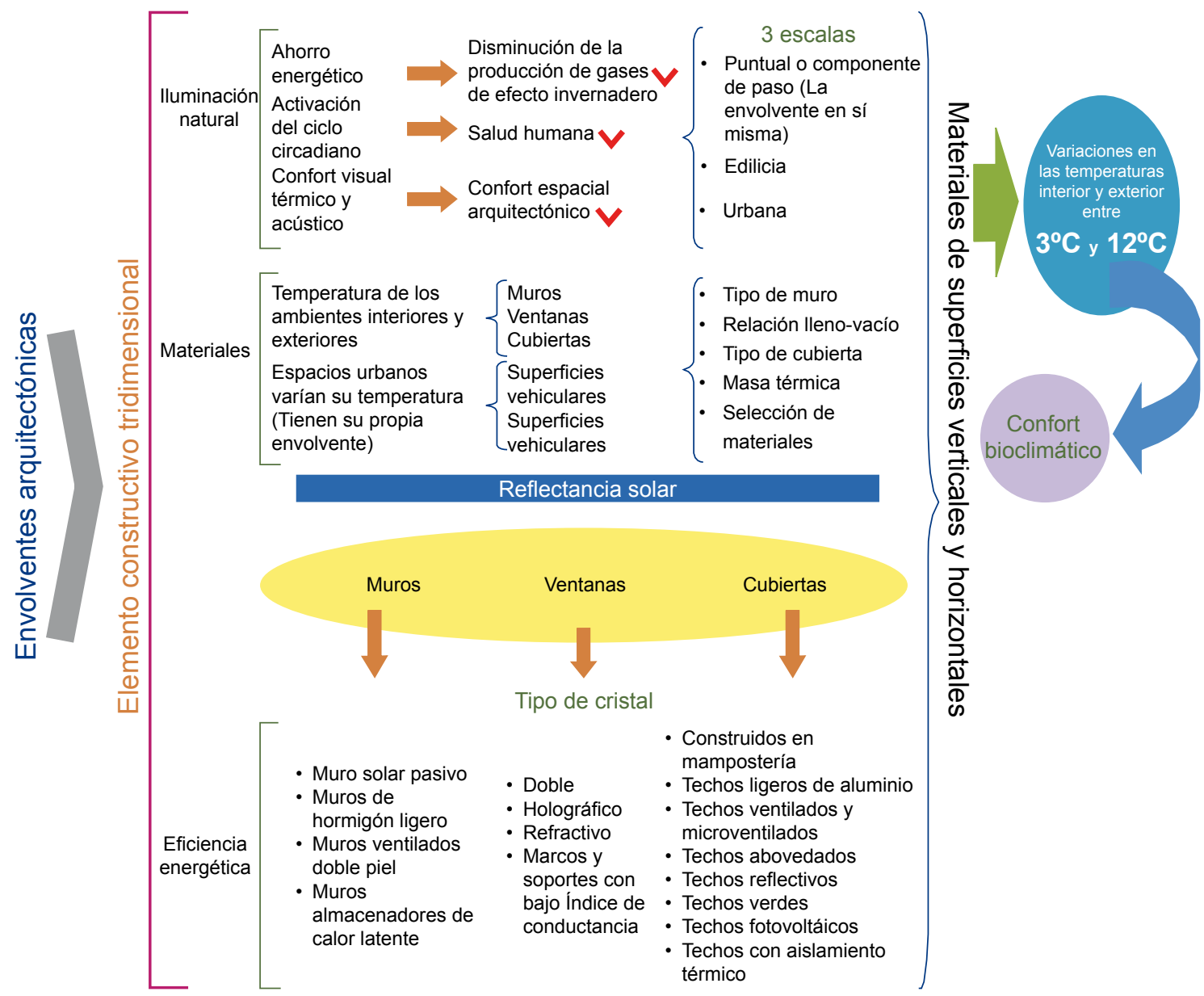

Figura 1. Síntesis para diseño y comprensión de envolventes arquitectónicas en edificaciones contemporáneas. 


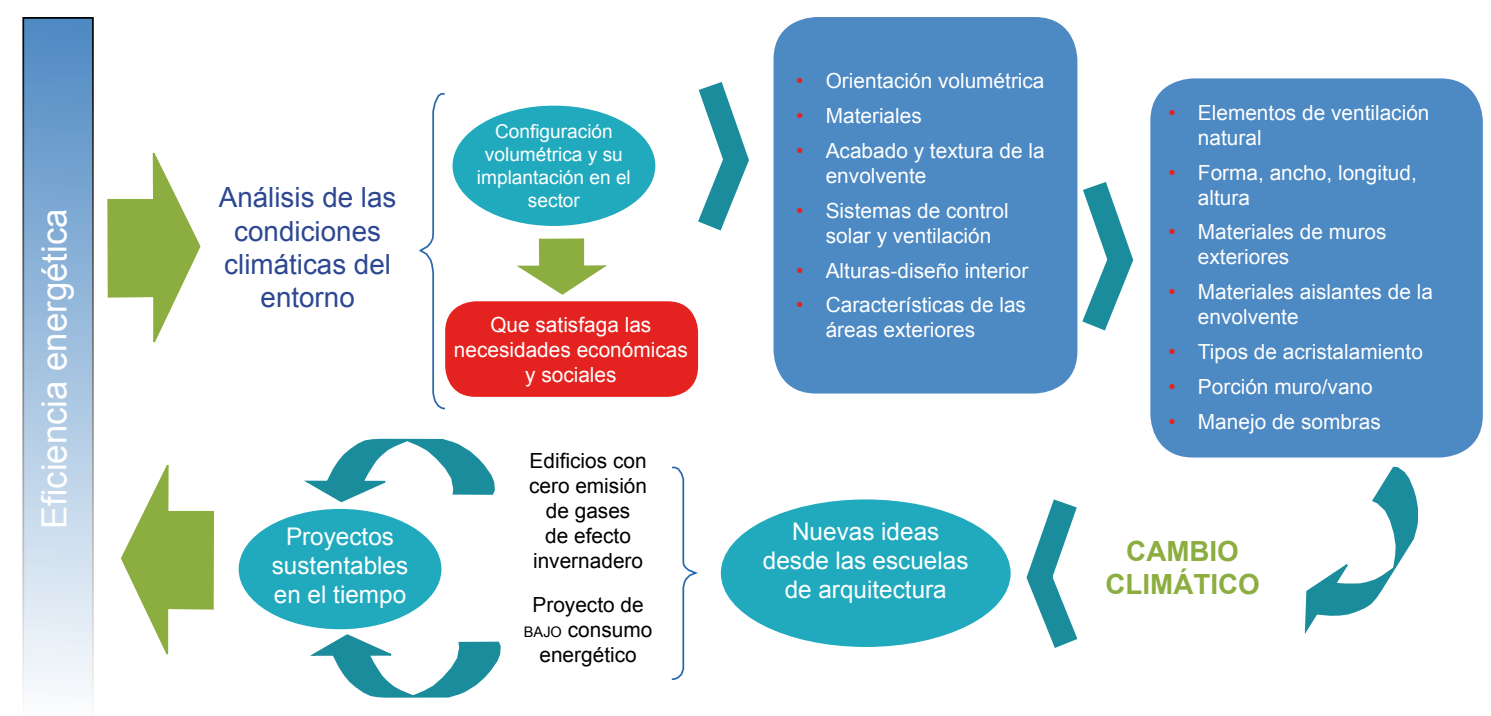

Figura 2. Síntesis gráfica de los resultados: las envolventes contemporáneas y su incidencia en la búsqueda de la eficiencia energética.

\section{Conclusiones}

La envolvente es un dispositivo particular y distintivo de la arquitectura contemporánea. Su desarrollo surge en la posmodernidad y se consolida en el siglo XXI. Se trata de un elemento tridimensional que recubre los objetos arquitectónicos e incluso algunos elementos urbanos, en el cual convergen de manera significativa los intercambios de luz, aire y energía, entre los espacios interiores de las edificaciones y su entorno inmediato. El concepto de envolvente supera la sumatoria de fachadas y cubierta de las construcciones, y su diseño debe ser desarrollado de manera integral, de forma tal que actúe como un intercambiador que regule - a discreción del diseñador- los flujos de aire, luz y energía que acontecen entre el afuera y el adentro de la arquitectura, en armonía con las exigencias de confort térmico de los usuarios y en respuesta a las determinantes del clima local. Esenciales para el diseño de la envolvente son los materiales con los que se construye, así como la geometría, forma y dimensión de los vanos, puesto que estos dos componentes de la envolvente determinan los índices de transmisión, aislamiento o conducción de calor, además de las posibilidades de iluminación natural y renovación del aire de la edificación. La envolvente es un componente tridimensional de toda arquitectura que debe diseñarse con rigor y detalle, para que las edificaciones logren realmente la meta de la sustentabilidad, y contribuyan eficientemente a las estrategias de mitigación del calentamiento global tan necesarias en el presente siglo, al reducir la demanda de energía generada por las edificaciones.

\section{Referencias}

Acosta, I., Campano, M., y Molina, J. (2016). Window design in architecture: Analysis of energy savings for lighting and visual comfort in residential spaces. Applied Energy, 168, 493-506.

Ackermann, M. (2013). Cool Comfort: America's Romance with Air-Conditioning. Washington, EE. UU.: Smithsonian Institution Press. 
Alchapar, N., Correa, E., y Cantón, M. (2013). Influencia del envejecimiento de los materiales en su desempeño térmico: El caso de revestimientos texturados para fachadas. Revista Latinoamericana de Metalurgia y Materiales, 33(2), 282-291.

Alchapar, N., y Correa. E. (2015). Reflectancia solar de las envolventes opacas de la ciudad y su efecto sobre las temperaturas urbanas. Informes de la Construcción, 67(540), 1-12.

Alonso, C., Oteiza, I., y García, J. (2010). Criterios para la reducción de emisiones de gases de efecto invernadero en el proyecto de fachadas de edificios de viviendas. En Actas II Congreso Nacional de Investigación y Edificación, Escuela Universitaria de Arquitectura Técnica, Universidad Politécnica de Madrid, Madrid.

Ávila, D., y Arias, S. (2015). La envolvente arquitectónica y su influencia en la iluminación natural. Revista Hábitat Sustentable, 5(1), 44-53.

Bahamon, A., y Cañizares, A. (2008). Iglú. De arquitectura vernácula a contemporánea. Madrid: Parramón.

Bennett, J. (2016). Global Ecological Crisis: Structural Violence and the Tyranny of Small Decisions. En Addressing Global Environmental Challenges from a Peace Ecology Perspective. (vol. 4 of the series The Anthropocene: Politik-Economics-Society-Science, pp. 55-75). México: Springer.

Berggren, B., y Wall, M. (2013). Calculation of thermal bridges in (Nordic) building envelopesRisk of performance failure due to inconsistent use of methodology. Energy and Buildings, 65, 331-339.

Biswas, K., y Abhari, R. (2014). Low-cost phase change material as an energy storage medium in building envelopes: experimental and numerical analyses. Energy Conversion and Management, 88, 1020-1031.

Brown, L., Flavin, C., y French, H. (2000). La situación en el mundo: informe anual del Worldwatch Institute sobre el medioambiente y el desarrollo. Barcelona, España: Icaria Editorial.

Bustamante, D. (2014). La profundidad de la envolvente (tesis doctoral). Universidad Nacional de Colombia, Sede Medellín, Medellín, Colombia.

Butti, K., y Perlin, J. (1985). Un bilo dorado: 2500 años de arquitectura y tecnología solar. Madrid, España: Blume.

Cabeza, L., Castell, A., Barreneche, C., De Gracia, A., y Fernández, A. (2011). Materials used as PCM in thermal energy storage in buildings: a review. Renewable and Sustainable Energy Revierws, 15(3), 1675-1695.

Celis, F., Escorcia, O., Díaz, M., García, A., y Echeverría, E. (2012). Incidencia de la forma y envolvente en el desempeño térmico de las viviendas del centro-sur de Chile. Hábitat Sustentable, 2(2), 19-33.

De Canales, F. (2001). Envolventes. DC PAPERS, Revista de Crítica y Teoría de la Arquitectura, 5-6, 62-71.

Dong, B., Cao, C., y Lee, S. (2005). Applying support vector machines to predict building energy consumption in tropical region. Energy and Buildings, 37(5), 545-553.

Evans, J., Eguía, S., Pérez, A., y Evans, J. (2003). Evaluación de impacto del sol en envolventes vidriadas. Avances en Energías Renovables y Medio Ambiente, 7(1), 37-42. 
Evans, J., y Schiller, S. (2005). Rol de la Envolvente en la Edificación Sustentable. Revista de la Construcción, 4(1), 5-12.

Fathy, H. (1986). Natural energy and vernacular architecture. Chicago, EE. UU.: The University of Chicago Press.

Färe, R., Grosskopf, S., y Tyteca, D. (1996). An activity analysis model of the environmental performance of firms-application to fossil-fuel-fired electric utilities. Ecological Economics, 18(2), 161-175.

Feathers, J., Johnson, J., y Kembel, S. (2008). Luminescence dating of monumental stone architecture at Chavín de Huántar, Perú. Journal of Archaeological Method and Theory, 15(3), 266-296.

Granadeiro, V., Duarte, J., Correia, J., y Leal, V. (2013). Building envelope shape design in early stages of the design process: Integrating architectural design systems and energy simulation. Automation in Construction, 32, 196-209.

Granadeiro, V., Correia, J., Leal, V., y Duarte, J. (2013). Envelope-related energy demand: A design indicator of energy performance for residential buildings in early design stages. Energy and Buildings, 61, 215-223.

Goia, F., Perino, M., Serra, V. y Zanghirella, F. (2010). Towards an active, responsive, and solar building envelope. Journal of Green Building, 5(4), 121-136.

Guzowski, M. (2013). Time and Adaptive Comfort Studies: Luminous and Thermal Design for Zero-Energy Architectural Education. En International Conference on Adaptation and Movement in Architecture, Toronto, Canada.

Heywood, H. (2015). 101 Reglas básicas para una Arquitectura de bajo consumo energético. Barcelona, España: Gili.

Janda, K. (2011). Buildings don't use energy: people do. Architectural Science Review, 54(1), 15-22.

Jie, F. (2014). Ecological crisis in postcolonial era: interpretation of disgrace from the perspective of colonialism and ecocriticism. Journal of Nanchang College of Education, 5. Recuperado de http://en.cnki.com.cn/Article_en/CJFDTOTAL-LCYY201405008.htm

Kantner, J. (2000). Realism vs. reality: creating virtual reconstructions of prehistoric architecture. $B A R$ International Series, 843, 47-52.

Khudhair, A., y Farid, M. (2004). A review on energy conservation in building applications with thermal storage by latent heat using phase change materials. Energy conversion and management, 45(2), 263-275.

Kuznik, F., David, D., Johannes, K., y Roux, J. (2011). A review on phase change materials integrated in building walls. Renewable and Sustainable Energy Reviews, 15(1), 379-391.

Lei, J., Yang, J., y Yang, E. (2016). Energy performance of building envelopes integrated with phase change materials for cooling load reduction in tropical Singapore. Applied Energy, $162,207-217$.

Lopez, M., Rubio, R., Martín, S., Croxford, B. y Jackson, R. (2015). Active materials for adaptive architectural envelopes based on plant adaptation principles. Journal of Facade Design and Engineering, 3(1), 27-38. 
Marincic, I., Ochoa, J., Alpuche, M., Duarte, A., Vargas, L., González, I., ... y Huelsz, G. (2011). La construcción actual de viviendas en Hermosillo y su adecuación al clima por medios pasivos. En Memorias de la XXXV Reunion Nacional de Energia Solar, ANES, Chihuahua, México.

Mirrahimi, S., Mohamed, M., Haw, L., Ibrahim, N., Yusoff, W., y Aflaki, A. (2016). The effect of building envelope on the thermal comfort and energy saving for high-rise buildings in hot-humid climate. Renewable and Sustainable Energy Reviews, 53, 1508-1519.

Méndez, T., Capozzoli, A., Cascone, Y., y Sassone, M. (2015). The early design stage of a building envelope: Multi-objective search through heating, cooling and lighting energy performance analysis. Applied Energy, 154, 577-591.

Moreno, S. (1993). Arquitectura, hombre y clima. Bogotá, Colombia: Universidad Nacional de Colombia/SENA.

Pérez-Lombard, L., Ortiz, J., y Pout, C. (2008). A review on buildings energy consumption information. Energy and Buildings, 40(3), 394-398.

Rubiano Martín, M. A. (2016). La fachada ventilada y el confort climático: un instrumento tecnológico para edificaciones de clima cálido en Colombia. DEARQ: Revista de Arquitectura de la Universidad de los Andes, 18, 138-145.

Sadineni, S. B., Madala, S. y Boehm, R. F. (2011). Passive building energy savings: A review of building envelope components. Renerwable and Sustainable Energy Revierws, 15(8), 3617-3631.

Santamouris, M., Papanikolaou, N., Livada, I., Koronakis, I., Georgakis, C., Argiriou, A., y Assimakopoulos, D. (2001). On the impact of urban climate on the energy consumption of buildings. Solar energy, 70(3), 201-216.

Sharma, A., Tyagi, V., Chen, C., y Buddhi, D. (2009). Review on thermal energy storage with phase change materials and applications. Renewable and Sustainable energy reviews, 13(2), 318-345.

Stevanovic, M. (1997). The age of clay: the social dynamics of house destruction. Journal of anthropological archaeology, 16(4), 334-395.

UNFPA (2015). Estado de la población mundial 2015. Nueva York: Fondo de Población de las Naciones Unidas.

Vela, F. (1995). Para una prehistoria de la vivienda. Aproximación historiográfica y metodológica al estudio del espacio doméstico prehistórico. Complutum, 6, 257-276.

Villalba, A., Pattini, A., y Córica, M. (2012). Análisis de las características morfológicas de las envolventes edilicias y del entorno urbano desde la perspectiva de la iluminación natural. Ambiente Construido, 12(4), 159-175.

Whitty, A. (1959). A classification of prehistoric stone buildings in Mashonaland, Southern Rhodesia. The South African Archaeological Bulletin, 14(54), 57-71.

Yan, H., Shen, Q. Fan, L. C., Wang, Y., y Zhang, L. (2010). Greenhouse gas emissions in building construction: A case study of One Peking in Hong Kong. Building and Environment, 45(4), 949-955.

Yang, L., Lam, J., y Tsang, C. (2008). Energy performance of building envelopes in different climate zones in China. Applied Energy, 85(9), 800-817.

Zhao, H., y Magoulès, F. (2012). A review on the prediction of building energy consumption. Renerwable and Sustainable Energy Reviews, 16(6), 3586-3592. 
